\title{
Electron Spin Resonance Spectroscopic Pursuit of Network Formation Processes in Free-Radical Crosslinking Polymerizations I. Microscopic Environmental Evaluation around Copper(II) Tetraphenylporphyrin Spin Probe Incorporated into or Excluded from Polymer Network in Methyl Methacrylate-Ethylene Dimethacrylate Copolymerization
}

\author{
Hiroyuki Aota, ${ }^{\dagger}$ Yasuyuki Sanal, Akira Matsumoto, ${ }^{\dagger}$ and Mikiharu Kamachi ${ }^{*}$ \\ Department of Applied Chemistry, Faculty of Engineering, Kansai University, Suita, Osaka 564, Japan \\ * Department of Macromolecular Science, Faculty of Science, Osaka University, \\ Toyonaka, Osaka 560, Japan
}

(Received March 4, 1996)

\begin{abstract}
The microheterogeneous network formation processes in the free-radical copolymerization of methyl methacrylate (MMA) with ethylene dimethacrylate (EDMA) were pursued Electron spin resonance (ESR)-spectroscopically by using copper tetraphenylporphyrin (CUTPP) and copper methacryloyloxyphenyl triphenylporphyrin (CuMAOTPP) as spin probes. Thus, CuTPP could be a useful tool to evaluate the mobility of low-molecular-weight molecules in the gelled system. In the MMA-EDMA copolymerization containing CuMAOTPP, three types of CuTPP moieties existed in the polymerization solution as immobilized in the densely crosslinked network, incorporated into the loosely crosslinked network, and unreacted monomer; their proportion was estimated by a semi-quantitative spectral simulation. For example, in the MMA-EDMA (70:30)-CuMAOTPP terpolymerization in a dilution of $1 / 10$ in benzene, $30 \%$ of CuMAOTPP was immobilized at $76 \%$ conversion by the densely crosslinked network or microgel, just like as in a frozen state, while no restricted mobility was observed for unreacted CuMAOTPP in spite of a complete loss of fluidity of the polymerization solution.

KEY WORDS Electron Spin Resonance / Copper Tetraphenylporphyrin / Network Formation / Crosslinking /Methyl Methacrylate / Ethylene Dimethacrylate /
\end{abstract}

For a long time, the network formation mechanism and the fine structure of cured resins have been controversial problems because of the complexity of the reactions involved and the insolubility of the products. In particular, the free-radical crosslinking polymerization and copolymerization of multivinyl compounds are the problem cases. Thus numerous mechanistic studies on the radical monovinyl-multivinyl copolymerizations accompanied by a three-dimensional network formation have been performed. ${ }^{1}$ On the basis of the comparison of the experimental results obtained mainly in our laboratory with Flory-Stockmayer $(\mathrm{F}-\mathrm{S})$ theory assuming ideal network formation, the greatly delayed gelation has been reasonably interpreted as follows: The primary factor is the thermodynamic excluded volume effect on the intermolecular crosslinking reaction between growing polymer radical and prepolymers, especially at higher molecular weight. Beyond the theoretical gel point, a secondary factor is the intramolecular crosslinking which becomes progressively important with conversion. The latter leads to the restriction of segmental motion of the prepolymer and, moreover, imposes steric hindrance, i.e., shielding effect or steric excluded volume effect, inducing the significance of the reduced reactivity as a tertiary factor.

In this connection, it is important to note that the occurrence of intramolecular crosslinking reaction is closely related to the formation of microheterogeneous network of the resulting crosslinked polymer, since the mechanical properties of crosslinked polymer significantly depend on the microheterogeneity of network structure. For example, the locally extensive occurrence of the intramolecular crosslinking leads to the formation

\footnotetext{
$\uparrow$ To whom correspondence should be addressed.
}

of microgel up to the gel point conversion ${ }^{2-5}$; here the microgel is conceived as consisting of both core and shell parts of high and low crosslinking densities, respectively, although it is soluble due to a strong interaction of the shell part with solvent overcoming the presence of the core part, just like a microsolid, having quite a weak interaction with the solvent. The homopolymerization of ethylene dimethacrylate (EDMA) and its copolymerization with methyl methacrylate (MMA) were the cases. However, no microgelation was observed in the bulk polymerization of diallyl phthalate (DAP) giving a quite low primary chain length ${ }^{6}$ and also in the monovinyl-divinyl copolymerization with only a small amount of divinyl monomer. ${ }^{7}$

In general, much information for the microheterogeneous network formation process is obtained, for example, by pursuing the change of the dependence of the radius of gyration of prepolymer on the molecular weight by using indirect ${ }^{3}$ and direct $^{4,5}$ light scattering methods. However, the pursuit of the process of growth of the gel with the progress of polymerization beyond the gel point has not been clarified yet because of poor methodology except for the bulk polymerization of $\mathrm{DAP}^{6}$ in which ester crosslinkages of the resulting gel are easily hydrolyzable.

Thus, we planned to pursue the process of network formation by electron spin resonance (ESR) spectroscopy, especially focusing on the microheterogeneity of network structure, since the segmental motion will be changed between the densely and loosely crosslinked network structures and ESR method has an advantage to study microscopic environments. In this connection, some ESR studies for network polymers have been reported from different standpoints: Hamielec group ${ }^{8,9}$ studied conformations, environments, and reactivities of 
growing polymer radical in the bulk copolymerization of MMA with EDMA accompanied by gelation. Schlick group $^{10}$ investigated the phase structure of the semiinterpenetrating polymer network (IPN) consisting of a crosslinked polyurethane and poly(vinyl chloride) by using spin probes. Also, they studied ESR spectra of hydrated $\mathrm{Cu}^{2+}$ in water-swollen polyacrylamide gels by chemical crosslinking and by $\gamma$-irradiation. In these studies, three types of the paramagnetic species were used: (1) growing polymer radical, ${ }^{8,9}$ (2) organic spin probes such as 2,2,6,6-tetramethylpiperidine-1-oxyl derivatives, ${ }^{10}$ and (3) metallic spin probes such as copper(II) ion. ${ }^{11}$ However, in the solution radical monovinyl-multivinyl copolymerization typical of the polymerization system accompanied by gelation, it is difficult to pursue the network formation process by using the above paramagnetic species because, in general, the concentration of growing polymer radicals in the solution radical polymerization is too low to detect them by ESR and moreover, organic and metallic spin probes essentially inhibit the radical polymerization. Thus, if there were a spin probe showing no influence on the radical polymerization, the spin probe would become a useful tool to study the network formation process of the solution radical crosslinking polymerization, especially focusing on the microheterogeneous network formation.

Here it should be noted that Kamachi et al. ${ }^{12}$ have reported the radical polymerization of vinyl monomers containing copper(II) tetraphenylporphyrin (CuTPP) moiety having the possibility of no inhibitory spin probe. On the other hand, Pezeshk et al. ${ }^{13}$ have studied the motional properties of CuTPP spin probe in lipid bilayers, the ESR spectral change for CuTPP being correlated with the change of the microscopic environment around the probe.

Thus, we tried to pursue the microscopic environmental change around CuTPP spin probe with the progress of gelation in MMA-EDMA solution copolymerization as a typical monovinyl-multivinyl copolymerization system. Here we used two types of spin probes of CuTPP and methacrylate containing CuTPP (CuMAOTPP) as shown in Chart 1: the former probe will be excluded from polymer network and the latter be incorporated into polymer network with the progress of polymerization. Moreover, it is noteworthy that microgelation occurs in MMA-EDMA solution copolymerization $^{2-5}$ as a result of locally extensive occurrence of intramolecular crosslinking leading to a microheterogeneous network formation.

Chart 2 shows a conceptual illustration of the microscopic environments around the spin probes in MMAEDMA-CuMAOTPP terpolymerization system. Three types of CuTPP moieties would typically exist in the polymerization system as immobilized in the densely crosslinked network, incorporated into the loosely crosslinked network, and unreacted CuMAOTPP monomer. Strictly speaking, the CuMAOTPP unit incorporated into the polymer chain is called "spin label" and the unreacted CuMAOTPP is "spin probe"; here we use, however, these terms indiscriminately as spin probes. The change of the motional property of CuMAOTPP spin probes incorporated into the polymer chain will reflect the microheterogeneity of polymer network, while the unreacted CuMAOTPP, similar to CUTPP, will act as a measure of the change of cavity of polymer network where small molecules in the polymerization system such as a residual monomer and solvent can move freely.

\section{EXPERIMENTAL}

\section{Materials}

MMA was washed with $5 \%$ of aqueous sodium hydroxide and distilled under reduced pressure. EDMA was purified by vacuum distillation. Benzene and toluene were commercially available products of super specific and specific grades, respectively. $N, N^{\prime}$-Azobisisobutyronitrile (AIBN) as an initiator was recrystallized from methanol. CuTPP was prepared according to the literature. ${ }^{14}$ CuMAOTPP was synthesized in the same method reported previously. ${ }^{12}$

\section{Polymerization}

Polymerization were carried out at $50^{\circ} \mathrm{C}$ in the similar manner reported previously. ${ }^{15}$

Poly(MMA-co-CuMAOTPP) was purified by reprecipitation from benzene-methanol system. A content of CuMAOTPP in the polymer was estimated from absorption spectrum in tetrahydrofuran (THF).

MMA homopolymerization and MMA-EDMA copolymerization in the presence of CuTPP or CuMAOTPP were carried out for ESR measurement as follows: For example, required amounts of MMA, EDMA, AIBN, and benzene were mixed. Then, one mg of CuTPP and

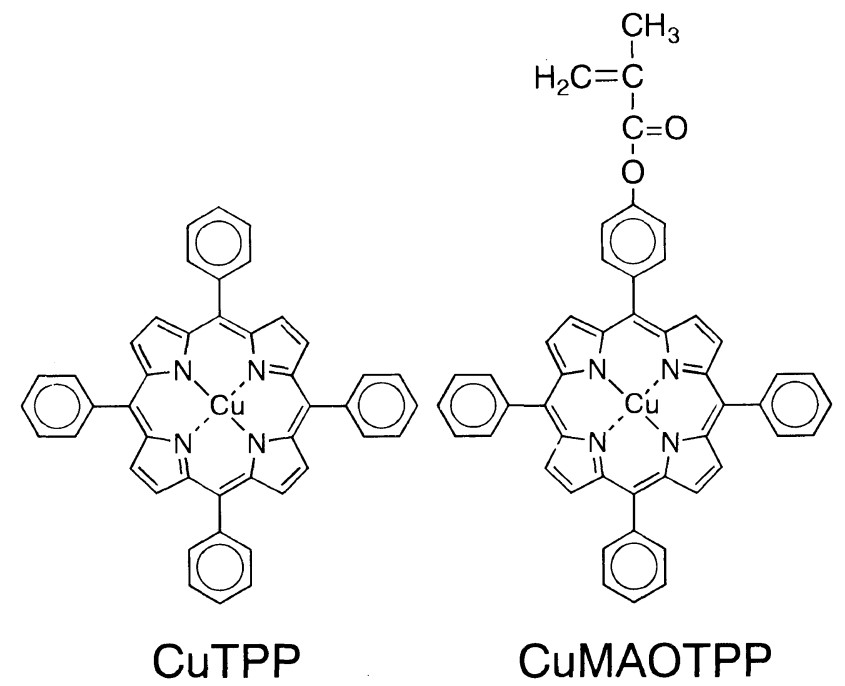

Chart 1.

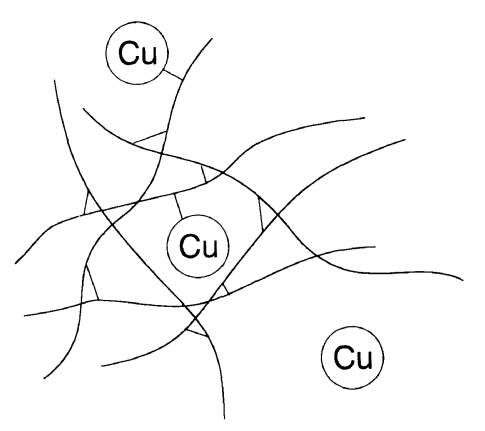

Chart 2. 
one $\mathrm{ml}$ of the benzene solution were put into a glass ampoule equipped with ESR tube of $5 \mathrm{~mm}$ diameter. The mixture was degassed on a vacuum line by five times freeze-pump-thaw cycles and then sealed under vacuum. The ampoule was then immersed in water bath controlled at $50 \pm 0.1^{\circ} \mathrm{C}$. The conversions of total resulting polymers and gel fractions were estimated ${ }^{16}$ from the separate polymerization under the same conditions as the above cases for ESR measurements.

\section{ESR Measurement}

$\mathrm{X}$-Band ESR measurements were performed on JEOL FE-1X spectrometer equipped with temperature control system under the following conditions: sweep width, $300 \pm 50 \mathrm{mT}$; modulation, $0.5 \mathrm{mT}$; microwave power, $2 \mathrm{~mW}$; and amplitude, moderate (100-10000). ESR data acquisition and spectral simulation were carried out on Macintosh Quadra 800 personal computer by using our program constructed based on LabVIEW2 (National Instruments Corporation).

\section{RESULTS AND DISCUSSION}

\section{Influence of CUTPP and CUMAOTPP Spin Probes on} Radical Polymerizability of $M M A$

In general, copper(II) ion inhibits the radical polymerization of vinyl monomers. Kamachi et al. ${ }^{17}$ have found that the radical polymerizabilities of MMA and styrene depended on the redox potentials of metalloporphyrins added into polymerization systems. Thus, $\mathrm{Fe}(\mathrm{III}) \mathrm{Cl}, \mathrm{Co}(\mathrm{II})$, and $\mathrm{Mn}(\mathrm{III}) \mathrm{Cl}$ tetraphenyl porphyrin complexes inhibited the polymerizations of MMA and styrene, but no inhibition occurred in the presence of CuTPP.

Here, the polymerizations of MMA in the presence of CuTPP or CuMAOTPP spin probes were carried out. Figure 1 shows the conversion-time curves; no significant difference was observed in the rates of polymerization between with and without spin probes. The molecular weights of the resulting polymers were essentially same. Thus, no inhibition of the radical polymerization of MMA due to CuTPP and CuMAOTPP was confirmed.

Contents of CuMAOTPP in poly(MMA-co-CuMAOTPP) polymerized for $4 \mathrm{~h}, 8 \mathrm{~h}$, and $16 \mathrm{~h}$ were 0.261 , 0.256 , and $0.252 \mathrm{~mol} \%$, respectively. This result indicates

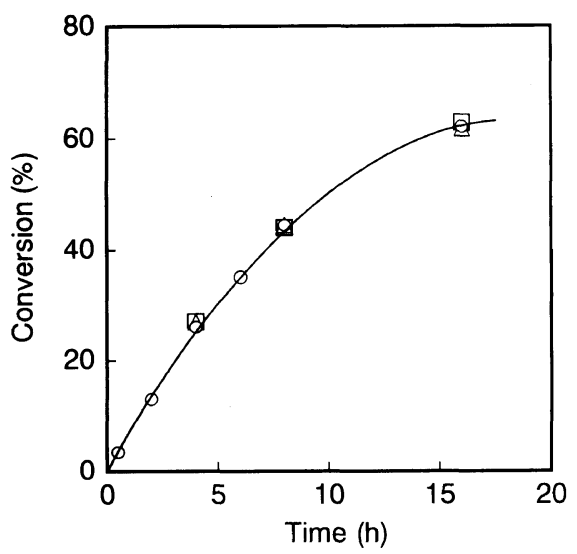

Figure 1. Conversion-time curves for the solution polymerizations of MMA in the absence or presence of spin probes: in benzene, dilution $1 / 10,[\mathrm{AIBN}]=40 \mathrm{~mm}$, [spin probe $]=1.5 \mathrm{~mm}, 50^{\circ} \mathrm{C}:(\bigcirc)$ none; $(\triangle)$ CuTPP; ( $\square$ ) CuMAOTPP. that the copolymerizability of CuMAOTPP with MMA is good.

Pursuit of Spectral Change of CUTPP as Evaluation of Mobility of Low-Molecular-Weight Molecules in Gelled System

At an early stage of MMA-EDMA copolymerization, the precopolymer having pendant double bonds is formed. The crosslinking reaction between the pendant double bond of the precopolymer and the growing polymer radical gradually becomes more important with the progress of polymerization and, eventually, gelation occurs and the viscosity of the system suddenly increases sharply. This drastic viscosity change attributed to the formation of a three-dimensional network of indefinitely large size is interesting by considering Pezeshk's study ${ }^{13}$ of a correlation of ESR spectral change with motional properties of CuTPP in light paraffin oil that ESR spectra of CUTPP varied drastically with temperature as a reflection of the fluidity change of light paraffin oil. Thus, in a gelled system, the mobility of CuTPP may be quite suppressed and the change of CuTPP mobility seems to be reflected on the ESR spectra.

So the change of CuTPP mobility was pursued as a model of the unreacted CuMAOTPP shown in Chart 2.

First, we took up MMA-EDMA $(70: 30$ by mol) copolymerization in the presence of CuTPP spin probe. Figure 2 shows ESR spectra of the copolymerization solutions at a dilution of $1 / 10$ in benzene (a) before polymerization and (b) at a total polymer conversion of $76 \%$ (gel content: $95 \%$ ), where ESR measurements were performed at $25^{\circ} \mathrm{C}$. No significant difference was observed between these spectra although the fluidity of the polymerization solution was completely lost by gelation at $76 \%$ conversion; no ESR spectral change of CuTPP regardless of the loss of a macroscopic fluidity caused by a network formation clearly demonstrates no mobility change of CuTPP molecule as the model of the unreacted CuMAOTPP. By a further consideration of this result, the microscopic mobility of the smaller molecules than CuTPP such as a residual monomer and a solvent would be unchangeable in this polymerization system during the progress of network formation.

Here it should be recalled that Pezeshk et al. ${ }^{13}$ have reported the suppressed mobility of CuTPP at the same temperature of $25^{\circ} \mathrm{C}$. However, the motion of CuTPP

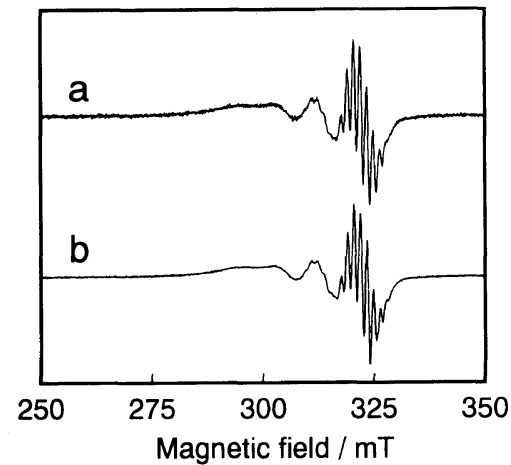

Figure 2. ESR spectra of MMA-EDMA(70:30) copolymerization solutions in the presence of CuTPP spin probe: (a) before polymerization; (b) at $76 \%$ conversion. Copolymerizations were conducted in benzene in a dilution of $1 / 10$ for $4 \mathrm{~h}$ using $40 \mathrm{mM}$ of $\mathrm{AIBN}$ at $50^{\circ} \mathrm{C}$ in the presence of $1.5 \mathrm{mM}$ of CuTPP. 
in this gelled system of a complete loss of fluidity is interestingly more active than that in light paraffin oil as judged by ESR spectral data. ${ }^{13}$ This kind of no ESR spectral change during crosslinking polymerization seems to be caused by the exclusion of the CuTPP molecules from the densely crosslinked polymer network such as a microgel and in addition, the low occupied volume of the resulting gel which is only about $7 \%$ for the total volume of this polymerization solution at the conversion $76 \%$. The latter factor prompts us to examine how the volume ratio of the network polymer to the small molecules such as solvent and residual monomer is needed to restrict the mobility of CuTPP excluded from the polymer network.

Figure 3 shows ESR spectra of MMA-EDMA (90: 10 by mol) copolymerization solution at a dilution of $1 / 2$ in benzene in the presence of CuTPP spin probe. Significant spectral change appeared above $70 \%$ conversion. This indicates that the high concentration of polymer $(\mathrm{ca} .35 \%)$ in the polymerization solution is required for restricting the motion of CuTPP. The required polymer concentration is also related to the microheterogeneity of network structure, although the detail will be discussed elsewhere.

\section{Pursuit of Spectral Change of CUMAOTPP as Evaluation of Microheterogeneous Network Formation}

As illustrated in Chart 2, the microscopic environments around the spin probes in MMA-EDMA-CuMAOTPP terpolymerization system are typically differentiated into three types. Here we will discuss the differentiation of two types of CuTPP moieties which are immobilized in the densely crosslinked network and incorporated into the loosely crosslinked network, re-

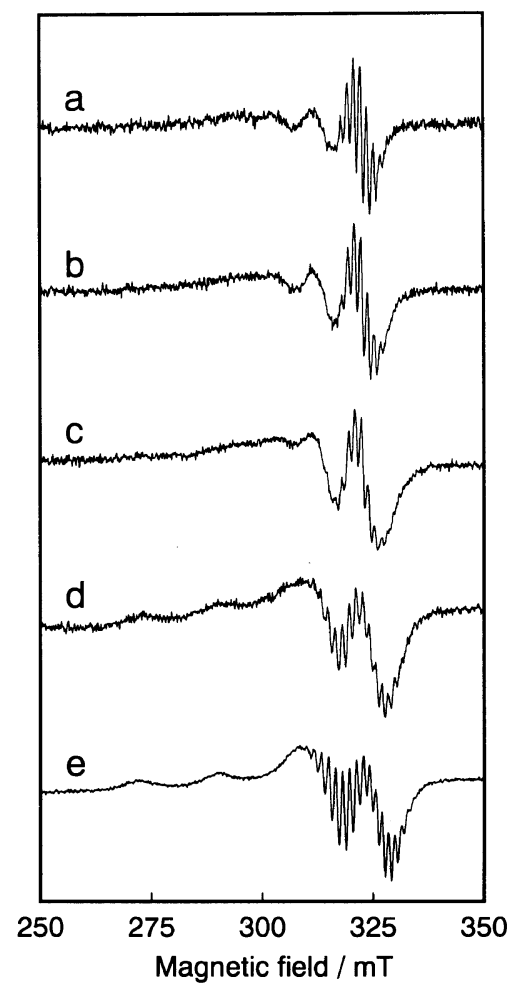

Figure 3. ESR spectra of MMA-EDMA(90: 10)-CuTPP system with a dilution of $1 / 2$ in benzene: conversion (\%), gel content (\%); (a) 0,0 ; (b) 28,97 ; (c) 74,99 ; (d) 97,97 ; (e) 99,96 . spectively, although the third type of CuTPP moiety as unreacted monomer was mentioned above.

Firstly, we examined the ESR spectral change of CuMAOTPP units incorporated into the MMA homopolymer chain as a model of the loosely crosslinked network. Thus, the copolymerization of MMA with CuMAOTPP was conducted in benzene at $50^{\circ} \mathrm{C}$; the polymerization solutions at (a) zero and (b) $8 \mathrm{~h}$ reaction times and (c) the solution of the resulting polymer in toluene were subjected to ESR measurements.

Figure 4 shows their spectra; spectral broadening appeared drastically in Figure $4 \mathrm{c}$, resulting from the restricted mobility of CuMAOTPP unit incorporated into the MMA polymer chain as the model of the loosely crosslinked network. Spectral profile of Figure $4 \mathrm{~b}$ is broader than that of Figure 4a because this spectrum is based on unreacted and polymerized CuMAOTPP.

Secondly, we tried to evaluate the spectral feature of CuMAOTPP unit immobilized by a densely crosslinked network. Thus, MMA-EDMA(90:10)-CuMAOTPP terpolymerization was carried out in benzene at a dilution of $1 / 10$ at $50^{\circ} \mathrm{C}$. Figure 5 shows the ESR spectra of the polymerization solutions at (a) zero and (b) $8 \mathrm{~h}$ reaction times. Apparently, the hyperfine structure (hfs) around $325 \mathrm{mT}$ became broad and shifted to slightly higher magnetic field after polymerization. This spectral change is different from the change observed in MMACuMAOTPP system as a spin probe simply incorporated into the polymer chain. Thus this spectral difference seems to reflect the degree of the restriction of CuMAOTPP moieties as a spin probe immobilized in the densely crosslinked network.

Next, we tried to check this immobilized effect of CuMAOTPP spin probe; MMA-EDMA(70:30)-CuMAOTPP terpolymerization was conducted under the same conditions because in this terpolymerization system microgelation is facile to occur as compared with the previous one and thus, the extent of immobilization of

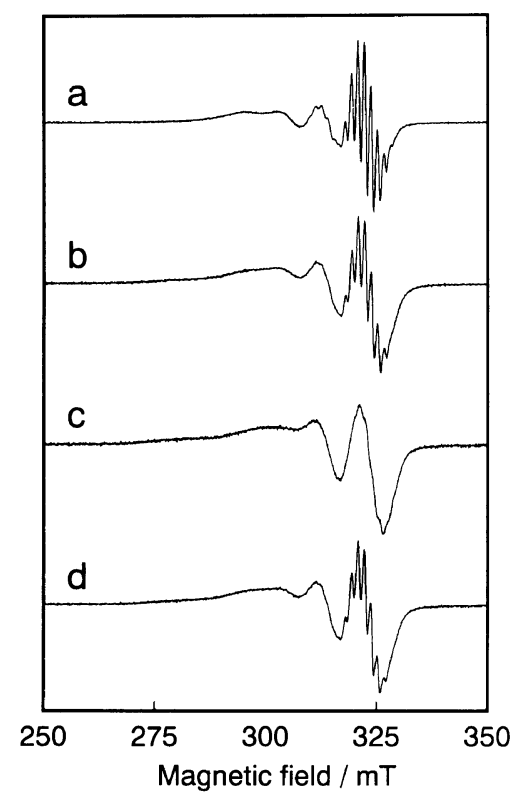

Figure 4. ESR spectra of MMA-CuMAOTPP system with a dilution of $1 / 10$ in benzene: (a) before polymerization; (b) at $44 \%$ conversion; (c) the solution of poly(MMA-co-CuMAOTPP) in toluene; (d) simulated spectrum. 


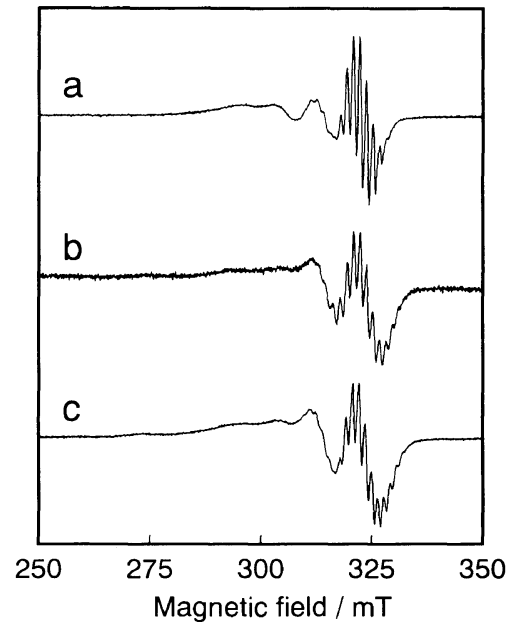

Figure 5. ESR spectra of MMA-EDMA(90:10)-CuMAOTPP system with a dilution of $1 / 10$ in benzene: (a) before polymerization; (b) at $66 \%$ conversion; (c) simulated spectrum.

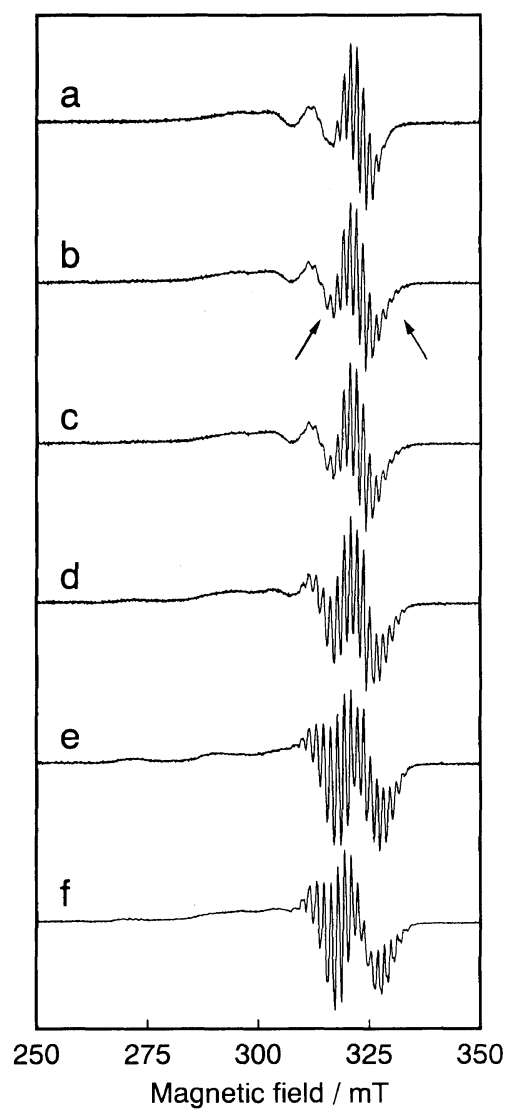

Figure 6. ESR spectra of MMA-EDMA(70:30)-CuMAOTPP system with a dilution of $1 / 10$ in benzene: conversion (\%), gel content $(\%)$; (a) 0,0 ; (b) 14, 0; (c) 29, 28; (d) 52, 79; (e) 76,95; (f) simulated spectrum.

the spin probe will be higher. Figure 6 shows the ESR spectral change of the polymerization solution with conversion; here the sharp hfs appeared in the magnetic region from 310 to $335 \mathrm{mT}$ and the hfs was observed even before the gel point as was slightly observed at arrows in Figure 6b. We will discuss in detail this result later.

\section{Attempt to Estimate Extent of Microheterogeneity of} Polymer Network by Spectral Simulation

As mentioned above, ESR spectral changes were

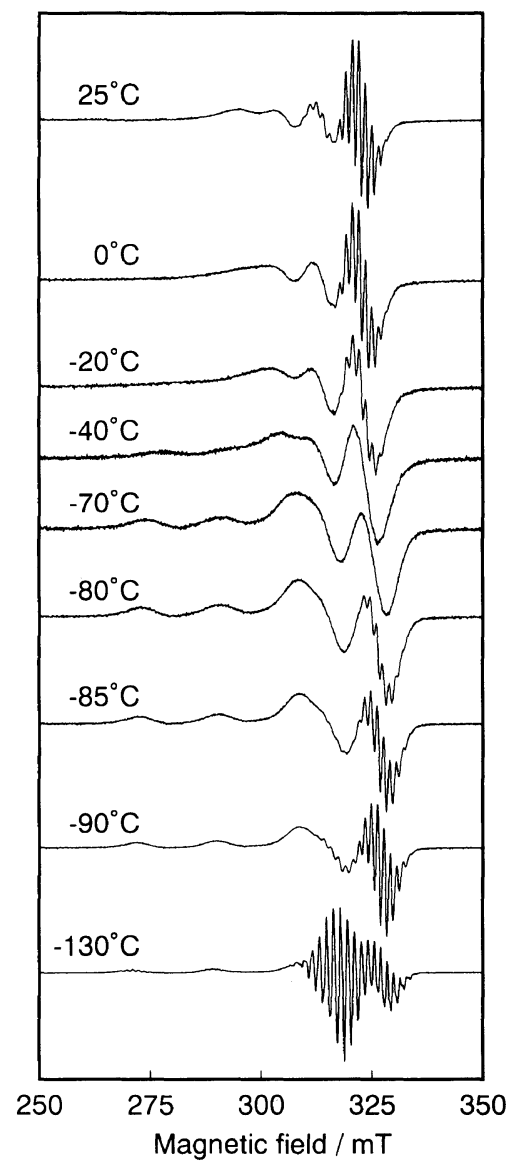

Figure 7. ESR spectra of $1.5 \mathrm{mM}$ CuTPP solution in toluene at different temperatures.

clearly observed with conversion. In order to estimate the microscopic environmental changes around the CuMAOTPP spin probe with the progress of network formation, we tried to simulate the ESR spectra semiquantitatively. In this connection, the ESR spectral changes of CuTPP in light paraffin oil at different temperatures have been studied ${ }^{13}$ and they are well correlated to the microscopic environmental change around CuTPP. However, the spectral change in the light paraffin oil is not suitable as a standard to estimate the microscopic environment around CuMAOTPP in our terpolymerization systems because CuTPP mobility in light paraffin oil ${ }^{13}$ is suppressed more strongly than that in benzene due to fluidity of solvent at the same temperature of $25^{\circ} \mathrm{C}$. So we chose the ESR spectral change of CuTPP in toluene at different temperatures (Figure 7) as the standard from the following two reasons: (1) ESR spectrum of CuTPP in toluene at $25^{\circ} \mathrm{C}$ was the same as that of the reaction mixture before polymerization and (2) a wide variety of ESR spectra of CuTPP were easily observable according to the extent of restriction of its mobility only by changing temperature from room temperature to $-130^{\circ} \mathrm{C}$ because of the lower melting point of toluene compared with benzene as the polymerization solvent. In Figure 7, the sharp hfs due to four nitrogen atoms in porphyrin ring was observed around $325 \mathrm{mT}$ at $25^{\circ} \mathrm{C}$; this demonstrates a rapid tumbling of CuTPP. By cooling the toluene solution, the gradual broadening of the hfs was observable up to $-70^{\circ} \mathrm{C}$, suggesting a suppression of the tumbling. Then, the sharp hfs reappeared at $-80^{\circ} \mathrm{C}$ and it became re- 
markable with lowering temperature although it shifted slightly to higher magnetic field; this should reflect the restricted mobility of CUTPP. ${ }^{13}$ Based on the standard spectra of the toluene solution of CuTPP at different temperatures as a reflection of the motion of CuTPP, we will discuss the ESR spectra of CuMAOTPP units shown in Figures 4, 5, and 6.

The spectrum of poly(MMA-co-CuMAOTPP) shown in Figure $4 \mathrm{c}$ is similar to that of CuTPP in toluene at $-40^{\circ} \mathrm{C}$. This indicates that the CuMAOTPP moiety incorporated into the polymer chain tumbles slowly as a result of the restricted motion. As a simplest case, we tried to simulate the ESR spectrum of $44 \%$ polymerization solution (Figure $4 \mathrm{~b}$ ) containing two types of CuTPP moieties belonging to either the unreacted CuMAOTPP monomer or the CuMAOTPP unit incorporated into the polymer chain. Since the content of CuMAOTPP in the MMA-CuMAOTPP copolymer obtained at conversion $44 \%$ was $0.256 \mathrm{~mol} \%, 75 \%$ of the feed CuMAOTPP monomer was incorporated into the polymer chain. Thus the simulated spectrum shown in Figure $4 \mathrm{~d}$ was based on a combination of $75 \%$ of the spectrum shown in Figure $4 \mathrm{c}$ and $25 \%$ of the spectrum of CuTPP toluene solution at $25^{\circ} \mathrm{C}$ shown on the top of Figure 7; it is quite similar to the observed one (Figure $4 \mathrm{~b}$ ). This simulation technique may be applicable to a rough estimation of the content of CuMAOTPP unit incorporated into the polymer chain in an unknown mixture of monomer and polymer.

As a more complex case, we tried to simulate the ESR spectrum of the $66 \%$ MMA-EDMA(90:10)-CuMAOTPP terpolymerization solution (Figure $5 b$ ) containing three types of CuTPP moieties belonging to unreacted CuMAOTPP monomer, the CuMAOTPP unit incorporated into the loosely crosslinked network, or

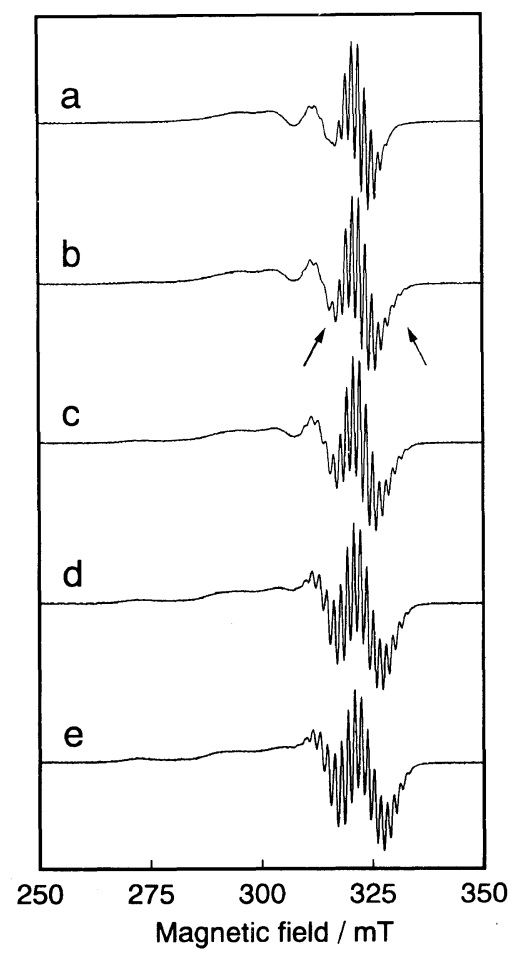

Figure 8. ESR spectra of MMA-EDMA(70:30)-CuMAOTPP system with a dilution of $1 / 20$ in benzene: conversion $(\%)$, gel content $(\%)$; (a) 0,0 ; (b) 36,0 ; (c) 48, 0; (d) 70, 0; (e) 74, 79 . the CuMAOTPP unit immobilized by the densely crosslinked network. The characteristic feature of the spectrum as shown in Figure $5 \mathrm{~b}$ was the shift to higher magnetic field of the hfs as observed similarly in the CuTPP toluene solution at $-85^{\circ} \mathrm{C}$ shown in Figure 7 ; this will be ascribable to the restricted mobility of CuMAOTPP unit immobilized by the densely crosslinked network. Since $19 \%$ of CuMAOTPP was remained as the unreacted unit at conversion $66 \%$, we can simulate the observed spectrum (Figure $5 \mathrm{~b}$ ) by a combination of $19 \%$ of the spectrum of CuTPP toluene solution at $25^{\circ} \mathrm{C}$ as a freely motional part, $x \%$ of the spectrum shown in Figure $4 \mathrm{c}$ as the units incorporated into the loosely crosslinked network without any motional restriction, and $y \%$ of the spectrum of the CuTPP toluene solution at $-85^{\circ} \mathrm{C}$ shown in Figure 7 as the units of restricted mobility, where $x+y=81$. By a trial and error method of changing $x$ and $y$, we reached the simulated spectrum similar to the observed one (Figure $5 b$ ) as shown in Figure 5c. Thus about $25 \%$ of CuMAOTPP units was immobilized by densely network.

Similarly, we tried to simulate the ESR spectrum of 76\% MMA-EDMA(70:30)-CuMAOTPP terpolymerization solution (Figure 6e) with our expectation of realizing the highly immobilized system; by a combination of $22 \%$ of the spectrum of the CuTPP toluene solution at $25^{\circ} \mathrm{C}, 48 \%$ of the spectrum shown in Figure $4 \mathrm{c}$, and $30 \%$ of the spectrum at $-130^{\circ} \mathrm{C}$, corresponding to the frozen state, shown on the bottom of Figure 7, we could depict the simulated spectrum by computer as shown in Figure 6f. From these simulations, we recognized that in the MMA-EDMA(70:30)-CuMAOTPP terpolymerization, the restriction of the motion of CuTPP moiety by the densely crosslinked network should be quite strong just like as in a frozen state. It should be also noted that the hfs around 317 and around $329 \mathrm{mT}$, which were observed under $-90^{\circ} \mathrm{C}$ of the CuTPP toluene solution shown in Figure 8 , appeared even before the gel point as is evident from Figure $6 \mathrm{~b}$. This may be relevant to the microgelation. In order to confirm this correlation, we examined a higher dilution terpolymerization system. Figure 8 shows the ESR spectra of MMA-EDMA $(70: 30)-C u M A O T P P$ terpolymerization system at a dilution of $1 / 20$. The strong restriction was clearly observed before the gel point conversion; no change appeared between just before $(70 \%)$ and after $(74 \%)$ gel point.

In this work, we showed that CuMAOTPP was a convenient spin probe and the spectral simulation was useful to estimate the change of the microscopic environment semi-quantitatively as the network formation proceeded in the solution radical copolymerization of MMA-EDMA. On the other hand, CuTPP spin probe is excluded from network, so the ESR spectral change of CuTPP system shows the change of cavity of polymer network. By using these spin probe methods, we will know the detail of network formation process beyond the gel point.

Acknowledgment. This work was partly supported by a Kansai University Research Grant. 


\section{REFERENCES}

1. A. Matsumoto, Adv. Polym. Sci., 123, 41 (1995) and references therein.

2. K. Dusek, "Developments in Polymerization," Vol. 3, Applied Science Publishers Barking, Essex, U.K., 1982, p 143.

3. A. Matsumoto, Y. Yamashita, and M. Oiwa, Netsukokasei Jushi (J. Thermoset. Plast., Jpn.) 12, 135 (1991).

4. A. Matsumoto, S. Takahashi, and M. Oiwa, Polym. Prepr., Am. Chem. Soc., Div., Polym. Chem., 31 (2), 149 (1990).

5. A. Matsumoto, Rep. Asahi Glass Found, 61, 187 (1992).

6. A. Matsumoto, H. Nakajima, and M. Oiwa, Netsukokasei Jushi (J. Thermoset. Plast., Jpn.) 9, 141 (1988).

7. A. Matsumoto, S. Okuno, and H. Aota, Makromol. Chem., Macromol. Symp., 93, 1 (1995).

8. S. Zhu, Y. Tian, A. E. Hamielec, and D. R. Eaton, Polymer, 31,
154 (1990).

9. Y. Tian, S. Zhu, A. E. Hamielec, D. B. Fulton, and D. R. Eaton, Polymer, 33, 384 (1992).

10. R. D. Harvey and S. Schlick, Polymer, 30, 11 (1989).

11. G. C. Rex and S. Schlick, J. Phys. Chem., 89, 3598 (1985).

12. M. Kamachi, X. S. Cheng, H. Aota, W. Mori, and M. Kishita, Chem. Lett., 2331 (1987).

13. A. Pezeshk, M. Pasenkiewicz-Gierula, W. K. Subczynski, and W. E. Antholine, J. Phys. Chem., 94, 451 (1990).

14. P. Rothemund and A. R. Menotti, J. Am. Chem. Soc., 70, 1808 (1948).

15. A. Matsumoto and M. Oiwa, J. Polym. Sci., A-1, 8, 751 (1970).

16. A. Matsumoto, T. Toyama, M. Oiwa, and M. Ochi, Netsukokasei Jushi (J. Thermoset. Plast., Jpn.), 12, 61 (1991).

17. X. S. Cheng, N. Watanabe, Y. Morishima, and M. Kamachi, Polym. Prepr. Jpn., 38, 1371 (1989) 